\title{
Creación e interpretación en el marco de los derechos de autor. El caso de la música popular tradicional
}

\section{Resumen}

El artículo muestra, utilizando el caso de la música popular tradicional, como los derechos de autor presentan limitaciones para la protección del trabajo creativo. A través de entrevistas a expertos y una revisión multidisciplinar de la literatura, se explica el proceso de creación de la obra y el papel de la interpretación en el mismo. Para ejemplificar lo anterior, utilizando datos obtenidos de entrevistas y encuestas, se presenta el caso representativo del flamenco. El principal resultado muestra como la interpretación es altamente valorada y, por tanto, se deja patente la brecha existente entre su valor percibido y la protección que recibe por parte de los derechos de autor.
Jesús Heredia-Carroza PhD. (c) Doctorando en Ciencias Económicas Universidad de Sevilla, España

Correo electrónico:

jhercar12@gmail.com

다이. orcid.org/0000-0003-2280-2680

Google Scholar

Luis Palma Martos, PhD.

Doctorado en Ciencias Económicas y Empresariales

Universidad de Sevilla, España

Correo electrónico: Ipalma@us.es

๑ o orcid.org/0000-0001-5834-3629

Google Scholar

Luis F. Aguado, PhD.

Doctorado en Economía

Pontificia Universidad Javeriana, Cali Cali, Colombia

Correo electrónico:

Ifaguado@javerianacali.edu.co

다이.org/0000-0002-1675-0418

Google Scholar

Recibido: agosto 28 de 2018

Aprobado: mayo 16 de 2019

Palabras clave:

Derechos de autor, enfoque multidisciplinar, flamenco,

interpretación, valor cultural. 


\section{Creation and performance in the framework of copyright. The case of traditional popular music}

\begin{abstract}
Using the case of traditional popular music, the article shows how copyright presents limitations in the protection of creative work. The process of creating the work and the role of interpretation are explained through interviews with experts and a multidisciplinary literature review. To illustrate the above, the representative case of flamenco is presented using data collected from interviews and surveys. The main result shows how interpretation is higly valued and therefore, the gap between its perceived value and the protection it receives from copyright is made clear.
\end{abstract}

Key words:

Copyright, cultural value, flamenco, performance, multidisciplinary analysis. 


\section{Introducción}

Es una realidad que las legislaciones internacionales de propiedad intelectual proporcionan una mayor cobertura jurídica a la composición realizada por los autores que a la interpretación llevada a cabo por los intérpretes. ¿Cómo explicar esta situación? Pareciera que la interpretación, desde el punto de vista de los diferentes ordenamientos jurídicos en materia de propiedad intelectual, es un trabajo rutinario (Caves, 2003), no creativo y de menor valor cultural (Bourdieu, 1984; Klamer, 2003; Heredia-Carroza et al., 2020) que la composición. El caso de la música popular tradicional ejemplifica lo anterior (Harkins, 2012, Heredia-Carroza et al., 2019c)).

La música popular tradicional tiene características estéticas, performativas y socioculturales propias, como son determinadas tendencias artísticas enfrentadas (pureza versus fusión) (Aix Gracia, 2002; Heredia-Carroza et al., 2019b), la importancia del entorno local o la mezcla de culturas (Romero, 1996; Guerrero, 2016). Su repertorio se caracteriza por una marcada tradición oral (Prouty, 2006) y una continua evolución, lo que permite considerar a muchos como géneros musicales vivos (Bermúdez \& Pérez, 2009).

La formación de sus músicos tradicionalmente ha sido empírica (Donnier, 2011; Heredia-Carroza et al., 2019a) en sus manifestaciones artísticas: danza, canto, música instrumental, entre otras (Blacking, 1981; Palma et al., 2017). La transmisión de las obras se aprende de otros directamente o de las grabaciones existentes y es raro que se utilicen partituras (McQueen \& Peacock, 1995; Donnier, 2011). De aquí se desprende la importancia de la interpretación (Edinborough, 2012; Heredia-Carroza, 2019); en palabras de Reyes (2013b) en la ejecución en vivo, el músico asume un rol crucial por encima de lo escrito y memorizado, se evidencia la necesidad de descubrir y entender las aportaciones que puede realizar un intérprete. 
El argumento principal del artículo sostiene que el intérprete en el desarrollo de la interpretación agrega aspectos a la obra, como son la improvisación, la espontaneidad e intuición artística (Romero, 1996; Cruces, 2001; HerediaCarroza et al., 2019b). Estas aportaciones se pueden considerar como un trabajo creativo que agrega valor cultural a la obra (Klamer, 2003; Edge, 2010; Duby, 2017; Heredia-Carroza et al., 2020). Sin embargo, dicho valor cultural no cuenta con una protección equivalente a su importancia por parte de los derechos de autor (Plant, 1934; Geiger, 2017; Giblin, 2017).

Para demostrar lo anterior, mediante entrevistas a personalidades de la industria musical española se contextualiza el papel de la interpretación dentro del proceso de creación de la obra. Seguidamente, se realiza una revisión bibliográfica multidisciplinar de: i. Artes escénicas, para confirmar la importancia que tiene la interpretación dentro del proceso de creación de la obra, ii. Derecho, para revisar el tratamiento legal que recibe el intérprete y cuáles son los requisitos que piden las legislaciones de propiedad intelectual para ser protegido por los derechos de autor. Por último, iii. Economía de la cultura, para diseñar una metodología que ayude a visualizar y a valorar las aportaciones realizadas por el intérprete en términos de valor cultural.

Para dar un ejemplo visible se presenta el caso del flamenco en España, género musical de origen popular tradicional que en 2010 fue aceptado en la Lista Representativa del Patrimonio Cultural Inmaterial de la Humanidad de la UNESCO (Palma et al., 2017; Heredia-Carroza et al., 2020). En el caso del flamenco se utilizarán datos recabados a partir de entrevistas en profundidad a artistas ${ }^{1}$ con trayectorias reconocidas y 690 encuestas a aficionados $^{2}(580)$ y

\footnotetext{
${ }^{1}$ El criterio de selección ha sido el currículum artístico de cada uno de ellos y que cuenten con la condición de ser autores además de intérpretes. ${ }^{2}$ Por aficionado se entiende a aquella persona que guiada por sus preferencias invierte sus recursos (tiempo, dinero) a través de su asistencia a espectáculos en vivo y compra de música grabada (Heredia-Carroza et al., 2019b).
} 
expertos $^{3}$ (110) para observar qué importancia le da el mercado a los aspectos ligados a la interpretación como son la improvisación, la espontaneidad y la intuición del artista.

El artículo constituye una aportación a la literatura conceptual y empírica sobre música popular tradicional. Concretamente, se diseña una metodología útil que permite medir la importancia creativa de la interpretación y muestra el desequilibrio generado por los derechos de autor, contando con las tres disciplinas involucradas: música, derecho y economía.

\section{Proceso de creación de la obra y papel de la interpretación}

La obra de música popular tradicional se considera un trabajo colaborativo entre autor e intérprete (Fernández, 2001; Webb, 2007; Gorton \& Östersjö, 2016; Heredia-Carroza et al., 2019c), sin embargo, esto no se desprende de lo recogido por las diferentes legislaciones en materia de propiedad intelectual. En el artículo se pretende mostrar empíricamente la brecha existente entre importancia de la interpretación y la protección proveída por dichas legislaciones.

Para ello, en primer lugar, se explica el proceso creativo y las fuentes de creatividad que aparecen en este. Esto se hace mediante entrevistas a expertos de reconocido prestigio de la industria musical española $y$, en segundo lugar, con bibliografía específica (Felton, 1978; Manuel, 2006; Towse, 2007; Pearson, 2010; Osborne, 2017), se contextualiza el papel de la interpretación dentro de dicho proceso de creación (Lumer, 1991). Las tres fuentes de creatividad son las siguientes (Pérez Peña, 2018; Heredia-Carroza et al., 2017):

\footnotetext{
3 Por experto se entiende aquella persona que a través de un entrenamiento especializado y de su experiencia acumulada (Holbrook, 1999), ya sea mediante el estudio o el ejercicio de su profesión, cuenta con un alto grado de conocimiento artístico (Bourdieu, 1984). En el artículo se considera expertos a los autores, intérpretes, críticos y gestores culturales.
} 
- Acervo Cultural: se corresponde con una fuente preexistente a las figuras del autor y del intérprete (Schiaffini, 2006), y de ella surgen las formas musicales, temáticas, patrones rítmicos y armónicos, entre otros (Scionti, 2017; Cruces, 2001; Deleuze y Guattari, 2002; Rosón, 2010; Manuel, 2010; Pérez Peña, 2018).

- Composición: se identifica con el trabajo creativo realizado por el autor de la obra (Throsby, 2016). En ella se unen los elementos derivados del acervo cultural y los creados por el autor, como son la letra y la melodía (Chojnacka, 2001; Manuel, 2006; Rosón, 2010).

- Interpretación: de ella se desprende la exteriorización de la obra, que es el momento en el que se completa su creación (Felton, 1978; González Betancur, 2013; Levinson, 2015; Heredia-Carroza et al., 2019b). En ella, se unen los elementos provenientes de la composición a los elementos propios del intérprete derivados de su talento y su concepto propio de los elementos provenientes del acervo cultural (Zavalloni, 2006; Hertzman, 2009) que permiten recrear lo preestablecido por el autor (Palmer, 1996; Towse 2007; Ordóñez Flores, 2011; Reyes, 2013b; Osborne, 2017; San Cristóbal Opazo, 2018; Heredia-Carroza et al., 2019c).

La figura 1 aclara el papel que desempeña cada fuente de creatividad de la obra musical y cómo se superponen en el proceso de creación. 


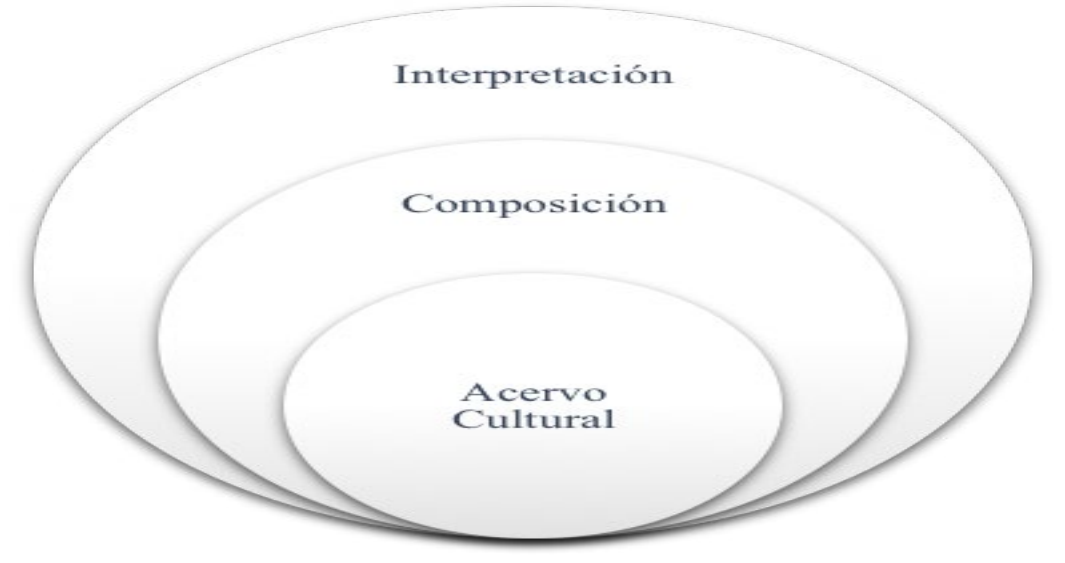

Figura 1. Fuentes de creatividad de la obra musical Fuente: Elaboración propia.

\section{Revisión multidisciplinar de la literatura}

Una de las aportaciones más importantes del estudio es su carácter multidisciplinar. La problemática tratada parte de lo musical, es causada por lo legal y para llegar a una solución necesita del marco metodológico de la economía de la cultura (Aguado et al., 2017; Monasterio-Astobiza, 2017; Heredia-Carroza et al., 2019c). Por ello, se realiza un estudio de la literatura propia de las artes escénicas, se estudian las diferentes regulaciones en materia de propiedad intelectual y, por último, se hace referencia a la bibliografía específica de economía de la cultura para diseñar la estrategia empírica a utilizar. 
Estudio de la literatura sobre artes escénicas

El título del artículo realizado por Pearson (2010) da una idea de lo que sucede en la actualidad con las aportaciones creativas de la interpretación: Authorship and Improvisation. Musical lost property. En él se deja patente como los elementos propios de la misma (improvisación, espontaneidad, intuición, entre otros) son una propiedad musical perdida para los intérpretes. De hecho, Pearson (2010) puntualiza que considera la necesidad de dar una mayor protección a la interpretación como algo único y no reproducible, por encima de la composición.

En esta línea, Chadabe (1999) defiende que la composición toma forma en cada actuación como resultado de un proceso mutuamente influyente entre el intérprete y su contexto. Chojnacka (2001) y Webb (2007) van más allá, considerando que compositores e intérpretes realizan un trabajo colaborativo en el proceso de creación artística (Heredia-Carroza et al., 2017).

Zavalloni (2006) considera que la libertad creativa es necesaria para que los intérpretes mediante la improvisación, espontaneidad e intuición recreen lo predefinido por los autores. En palabras de Edinborough (2012): "durante la interpretación los intérpretes moldean colectivamente la obra planificada con base en la ocurrencia espontánea no planificada" (p. 199).

McNeil (2017) defiende la idea del modelo de fixed seed ideas o ideas predefinidas fijas, que se moldean creativamente durante la interpretación. Este modelo proporciona un marco conceptual más relevante y apropiado para comprender los procesos de creación en géneros musicales con una marcada tradición oral. Es esta tradición oral lo que da a los géneros musicales una identidad única con respecto a otras formas de música que se describen como tradiciones escritas (Prouty, 2006). 
Thomas (2007) habla sobre la naturaleza de las decisiones de los intérpretes tanto antes como durante la interpretación (Gorton \& Ostersjö, 2016). Las cuestiones que definen las decisiones del artista sobre cuándo, cómo y qué interpretar se contextualizan tanto en lo marcado por el compositor como por las tradiciones performativas que el intérprete desarrolla durante su carrera. Defiende que la interpretación de músicas no escritas, se aleje de enfoques cerrados y estandarizados por la notación y los compositores, abriéndose así a nuevas posibilidades (Gorton \& Ostersjö, 2016).

De especial relevancia son los trabajos de Manuel (2006; 2010), que tratan el concepto de autoría en géneros musicales caracterizados por la tradición oral. Según Manuel, habría que crear nuevas concepciones y nociones de propiedad intelectual tal y como están incorporadas en los actuales regímenes de derechos de autor, a fin de llegar a una solución para la problemática descrita. En el siguiente apartado se muestran las posturas actuales de diferentes regímenes de propiedad intelectual para que un trabajo creativo pueda ser protegido por derechos de autor.

\section{Estudio de legislaciones de propiedad intelectual}

Según la Organización Mundial de la Propiedad Intelectual, en el documento La observancia de los derechos de propiedad intelectual (Rangel, 2011), los derechos ostentados por autores e intérpretes son:

- Los derechos de autor (Throsby, 2016) se centran en los derechos patrimoniales de reproducción, distribución, comunicación pública y transformación y los morales de paternidad, integridad, divulgación, modificación, retirada del comercio por cambio de convicciones intelectuales o morales y acceso a ejemplar único o raro (Rushton, 1998). 
- Los derechos para los intérpretes, denominados derechos vecinos o conexos (McQueen \& Peacock, 1995), no incluyen el derecho patrimonial de transformación, ni los morales de: divulgación, modificación, retirada del comercio por cambio de convicciones intelectuales o morales y acceso a ejemplar único o raro (Towse, 2007).

Un tema controvertido es la duración de los derechos patrimoniales (Harkins, 2012; Giblin, 2017), ya que los derechos de los autores duran toda su vida y 70 años después de su muerte o declaración de fallecimiento; no obstante, para los intérpretes duran 70 años desde la interpretación o divulgación lícita de la grabación de su ejecución (Directiva 2011/77/EU) ${ }^{4}$.

Una vez presentadas las diferencias entre los derechos que se le reconocen a los autores y a los intérpretes, se presentan qué requisitos imponen los diferentes ordenamientos jurídicos en materia de propiedad intelectual para ser cubiertos por los derechos de autor. Para ello se recurre al concepto de originalidad subjetiva (de Román, 2003; Heredia-Carroza et al., 2017).

- Variación distinguible (distinguishable variation ${ }^{5}$ ): requisito defendido por los tribunales estadounidenses, según el cual una persona puede ser considerada autor si realiza una variación apreciable con respecto a las obras preexistentes.

- Protección global de la obra: apunte extraído de sistemas anglosajones como el británico, australiano o neozelandés. Las contribuciones

\footnotetext{
${ }^{4}$ La Directiva 2011/77/EU es un paso muy importante para los intérpretes ya que aumentó la duración de sus derechos de 50 a 70 años desde la interpretación o divulgación lícita de la grabación de su ejecución. Esta directiva deja patente la estrategia europea de darle una mayor protección a la interpretación.

${ }^{5}$ Circular 92, Copyright Law of the United States and Related Laws Contained in Title 17 of the United States Code. Dec. 2012. Recuperado de: https://www.copyright.gov/title17/

Circular 96. Code of Federal Regulations, Title 37- Patents, Trademarks, and Copyrights, Copyright Office regulations codified in the Code of Federal Regulations (CFR). Recuperado de:

https://www.copyright.gov/title37/202/.
} 
pueden ser protegidas como obras autónomas, como puede ocurrir con la letra y la música. Sin embargo, se protege el conjunto de la obra en un sentido amplio (González, 2001).

- Impronta del autor: este concepto ha sido utilizado por la Cour de Cassation francesa. De él se desprende que la originalidad subjetiva depende del sello distintivo del autor, independientemente de si la obra es novedosa o no ${ }^{6}$.

- Altura creativa: requisito proveniente de la Persönliche geistige Shöpfungen alemana (de Román, 2003, p. 39). El ordenamiento jurídico alemán impone un nivel mínimo de novedad que puede ser fijado de forma distinta, dependiendo de la naturaleza de la obra y el grado de libertad creativa que se imponga (Reyes, 2013a).

- Novedad: criterio sostenido por las directivas comunitarias ${ }^{7}$ concernientes a propiedad intelectual (Pulido et al., 2016), no prestando tanta atención a la personalidad que pueda aportar el autor.

- Criterios artísticos, culturales, espirituales y técnicos: criterios sostenidos por la Constitución lituana de 1992 o las legislaciones serbia, venezolana o del Congo (Geiger, 2017).

Los criterios citados se basan en la originalidad subjetiva (Castillo Ballén, 2015), con lo que para medirlos se necesita de una metodología sólida que lo permita, por ello se recurre a la economía de la cultura.

\footnotetext{
${ }^{6}$ Sentencia de la Cour de Cassation de 1 de julio de 1970.

${ }^{7}$ Directiva 2001/29/CE, de 22 de mayo - LCEur $\backslash 2001 \backslash 2153$. DERECHO DE AUTOR. Armonización de determinados aspectos de los derechos de autor y derechos afines a los derechos de autor en la sociedad de la información y Directiva 2011/77/UE del Parlamento Europeo y del Consejo de 27 de septiembre de 2011 por la que se modifica la Directiva 2006/116/CE relativa al plazo de protección del derecho de autor y de determinados derechos afines. Diario Oficial de la Unión Europea, 10 de octubre de 2011.
} 
Estudio de la literatura sobre economía de la cultura

Caves (2003) acuñó el concepto hummdrum input, propio para trabajos rutinarios, no creativos y de bajo valor cultural. Una vez se han estudiado los diferentes regímenes de propiedad intelectual se puede observar cómo la interpretación está recibiendo un tratamiento de trabajo rutinario por parte de los mismos (Heredia-Carroza et al., 2019c).

Towse (2007) se refiere a la posibilidad de que el talento del intérprete pueda ser equivalente a la creatividad del autor. Sin embargo, en el mercado se produce un desequilibrio en la protección al trabajo creativo derivada de los instrumentos de protección citados. Además, defiende que la interpretación dota de rareza y belleza a la obra, haciéndola única.

Felton (1978) es uno de los autores clave en economía de la cultura para defender la interpretación como un trabajo creativo. En su trabajo, Felton habla de la necesidad de la interpretación para que la obra musical llegue al público, lo que posteriormente Heredia-Carroza et al. (2019a) definieron como la necesidad de la exteriorización de la obra para finalizar el proceso de creación.

Pitt (2010) y Meiseberg (2014) tratan el efecto que causa el artista que interpreta la obra en la recaudación de royalties o rendimientos económicos que genera la misma. Estos estudios son clave ya que demuestran como una obra interpretada por un intérprete $u$ otro puede causar diferentes niveles de impacto en el mercado.

Además de contextualizar la brecha creada por los derechos de autor, la bibliografía propia de la economía de la cultura se usa en este trabajo para diseñar una metodología que permita medir el valor cultural percibido de la interpretación (Bourdieu, 1984; Klamer, 2003; Icazuriaga et al., 2016; Hernando \& Campo, 2017; Osborne, 2017). 
Dicha metodología se basa en dos aproximaciones, por un lado, en entrevistas ${ }^{8}$ en profundidad a artistas, con la que se obtuvo información cualitativa. Por otro lado, en 690 encuestas de valoración realizadas a los agentes del mercado del flamenco (Hagtvedt et al., 2008; Radbourne et al., 2010; Au et al., 2016; Hadi Akdede \& Ogus Binatli, 2016; Hernando \& Campo, 2017; Lundy \& Smith, 2017; Chan et al., 2017).

\section{El caso del flamenco en España}

Para dar un ejemplo visible de la problemática expuesta durante el desarrollo del artículo, se presenta el caso del flamenco en España. El flamenco es un género de música popular tradicional cuyo origen se data en el s. XVIII (Infante, 1980; Romero, 1996; Steingress, 2007). Su riqueza y variedad se observan desde una doble perspectiva: por un lado, desde las culturas de las que tomó elementos -andaluza, gitana, árabe, judía- (Romero, 1996; Cruces, 2001); por otro lado, desde sus tres tipos de manifestaciones artísticas como son el cante -música vocal-, el toque -música instrumental- y el baile -arte de la danza(Infante, 1980; Manuel, 2010).

En el desarrollo de este epígrafe se presenta la información cualitativa obtenida a través de las entrevistas y, posteriormente se presenta la información cuantitativa conseguida a partir de las 690 encuestas a los agentes del mercado del flamenco.

\footnotetext{
${ }^{8}$ Su duración fue de entre 60 y 90 minutos y se llevaron a cabo en el periodo enero/mayo de 2017. Dichas personalidades son: autores que además son intérpretes de las tres manifestaciones del flamenco -cante, baile y toque-; políticos responsables del área de cultura -Exconsejero de Cultura de Andalucía-; representantes de las dos entidades de gestión de derechos de propiedad intelectual más importantes de España (Sociedad General de Autores y Editores y la Asociación de Artistas, Intérpretes y Ejecutantes); o gestores culturales de los festivales "Bienal de Flamenco de Sevilla" y del "Festival de Jerez de la Frontera". Los criterios de selección de los entrevistados fueron su relevancia, teniendo en cuenta los premios obtenidos en el caso de los artistas; los festivales que dirigen para los gestores culturales; o el nivel de responsabilidad en temas relacionados con la cultura.
} 


\section{Entrevistas}

Para medir la importancia de la interpretación y sus aportaciones a la obra musical flamenca se han entrevistado a varios de los artistas más importantes del panorama actual del flamenco. Todos ellos comparten la característica de ser autores e intérpretes de gran parte de las obras que presentan ante público.

El guitarrista Tomatito en su entrevista habló de que en la música no escrita no te manda nadie, si no hay nada predefinido, tú tienes una idea, una estructura y la moldeas según tu criterio artístico. Lo que está en consonancia con la idea de fixed seed ideas defendida por McNeil (2017).

José Mercé trató el tema de la performance: la performance depende totalmente del intérprete, el flamenco es un género musical que lo permite. El eco, el dolor, que la música hiera, te rompa, depende del intérprete. Además del trabajo predefinido del autor, es fundamental que el intérprete tenga gusto cantando, que sepa modular y añada elementos propios. Ahora bien, dichos elementos deben partir de una raíz, para ello hay que saber de dónde viene el flamenco, y a partir de la base predefinida que nos da el acervo cultural, crear.

Marina Heredia al ser preguntada sobre la importancia de la espontaneidad y de la intuición del artista dijo lo siguiente: Ese aspecto depende del estudio, la preparación, tu forma de estar ese día en el escenario. La espontaneidad e intuición dependen de todos los que estamos en el escenario, es muy importante y el flamenco se basa mucho en eso: sabes cómo empiezas, cómo acabas, pero no lo que pasa en medio de la actuación. En 2016 en la Bienal de Flamenco de Sevilla, con el espectáculo A mi tempo ${ }^{9}$, tuve que trabajar con seis guitarristas diferentes, con su historia y mundos propios. Tuve que amoldarme a ellos, de eso se trataba el espectáculo y era mi reto: nos entendimos totalmente. No fue un espectáculo donde se ensayara mucho, la 
idea era otra. Por ejemplo, con Paco del Gastor ${ }^{10}$ no ensayé, él me preguntó el tono, la velocidad, le canté un poco y a volar, no fue más. La intuición y la espontaneidad en la posterior presentación fue importantísimo, y todo esto se construye sobre la base marcada por el acervo cultural.

Rocío Márquez habló sobre el caso concreto de la interpretación de composiciones que no son propias. En estos casos modificas la melodía a tus habilidades, capacidades y a tu propio entendimiento del acervo cultural flamenco. Yo intento respetar la melodía pero lo llevo donde pueda defenderlo mejor a nivel vocal. También trató el tema de la improvisación en el flamenco: A mí me encantan los cantaores y cantaoras que improvisan, lo que también creo es que hay mito. Mito porque en los últimos años se ha caído en el enfoque academicista del flamenco y de la reproducción simple. Para mí improvisar no es coger patrones personales que te funcionan y ponerlos cada día en un sitio distinto. Es algo que has trabajado previamente en el estudio, ahí eres libre e improvisas. Un tema que está cerrado intentas cantarlo de otra forma. Esto es muy necesario para que el arte evolucione. Entiendo, que cuando existe un trabajo creativo por parte del intérprete, ya sea a nivel técnico, rítmico, melódico, este debería ser reconocido. Pero también creo que la gran mayoría no hace esto, a ese nivel se llega con el tiempo, es un proceso. Cuando empiezo un cante intento que sea lo más fiel posible a lo preestablecido y poco a poco vaya amoldándose a mí e ir añadiendo mis elementos propios. Por último, resaltó la importancia de lo individual: cada artista como ser único hará aportaciones únicas a las obras, es más, dependiendo del momento en el que lo escuches, estas aportaciones serán diferentes.

Para finalizar las entrevistas, el bailaor Barullo trató un ejemplo propio de una actuación donde la intuición y espontaneidad del cuadro flamenco fue

${ }^{10}$ Francisco Gómez Amaya, guitarrista gitano nacido en la localidad de Morón dela Frontera (Sevilla) en el año 1944. Para más información: https://elartedevivirelflamenco.com/guitarristas136.html. 
fundamental: ese día estaba especialmente cómodo improvisando al final de la actuación, los cantaores cantaban con mucho compás ${ }^{11}$, el público me estaba motivando, esto me metió en una espiral de la que no supe salir a su tiempo. Esta improvisación se estaba haciendo excesivamente larga y uno de los palmeros con una palmada hizo que tanto el cuadro $^{12}$, como yo paráramos justo en el mismo instante. Esa intuición que todos tuvimos con una sola palmada que sonó un poco más alta que otra fue increíble, en el momento no me di cuenta, pero a posteriori repasando la actuación en vídeo, observé la gran compenetración que existía en el equipo y el poder de la espontaneidad y la intuición: una palmada lo paró todo.

Como se puede observar de la información cualitativa obtenida a través de las entrevistas, se desprende la importancia de la figura del intérprete en el flamenco.

\section{Encuestas}

Para medir cuantitativamente la percepción del valor cultural de la interpretación y de los aspectos ligadas a la misma realizaron 690 encuestas, 580 a aficionados y 110 a expertos (51 a críticos y 59 a gestores culturales ${ }^{13}$ ). Se llevaron a cabo entre mayo y julio de 2017, a través de un instrumento de encuesta online dispuesto en Flama. La guía del flamenco ${ }^{14}$, que además contó con la divulgación del Centro Andaluz de Documentación del Flamenco y la web Promocionmusical.es. Se diseñaron dos instrumentos de encuesta, dirigido uno a los expertos y otro a los aficionados. Los instrumentos se dividieron en cuatro apartados. En el primer apartado, se preguntó por el perfil profesional de los expertos y de hábitos de consumo de flamenco de los aficionados. En

\footnotetext{
${ }^{11}$ Término usado en el flamenco para referirse al ritmo.

${ }^{12}$ Conjunto de artistas que realizan la actuación.

${ }^{13}$ Los cuestionarios y la base de datos están disponibles a solicitud a los autores.

${ }^{14}$ Mayor portal de información, venta de tickets, agenda de espectáculos y noticias sobre flamenco. Opera en España y Francia de manera

mensual desde enero de 2006. Para más información: https://www.guiaflama.com/.
} 
el segundo, se realizaron 41 preguntas de valoración de la obra flamenca. En el tercer apartado se realizan cuestiones sobre política cultural, con cuatro preguntas acerca de si al flamenco se debe apoyar con fondos públicos para su promoción. En el cuarto apartado, se indaga por los aspectos sociodemográficos básicos (p.ej. edad, sexo, nivel educativo). Los apartados 2, 3 y 4 fueron comunes para ambos grupos.

Para la presente investigación, siguiendo la línea argumental marcada por las entrevistas, se han tomado las respuestas de valoración de las aportaciones ligadas a la interpretación y a la improvisación (Rosón, 2010; Romero, 1996).

A continuación se presentan dos gráficos donde se podrá observar la valoración que recibe tanto la interpretación como los elementos ligados a la improvisación. Ambos están divididos en cuatro niveles de valoración: no sabe/no contesta, bajo, indiferente y alto.

En el caso de la valoración de la interpretación, se observa claramente como la valoración de la misma dentro de la obra musical, tanto por parte de los aficionados como de los expertos, es alta. Concretamente, más de un $72 \%$ de ambas categorías perciben que la interpretación desempeña un papel importante en términos de valor cultural para la obra musical flamenca. 


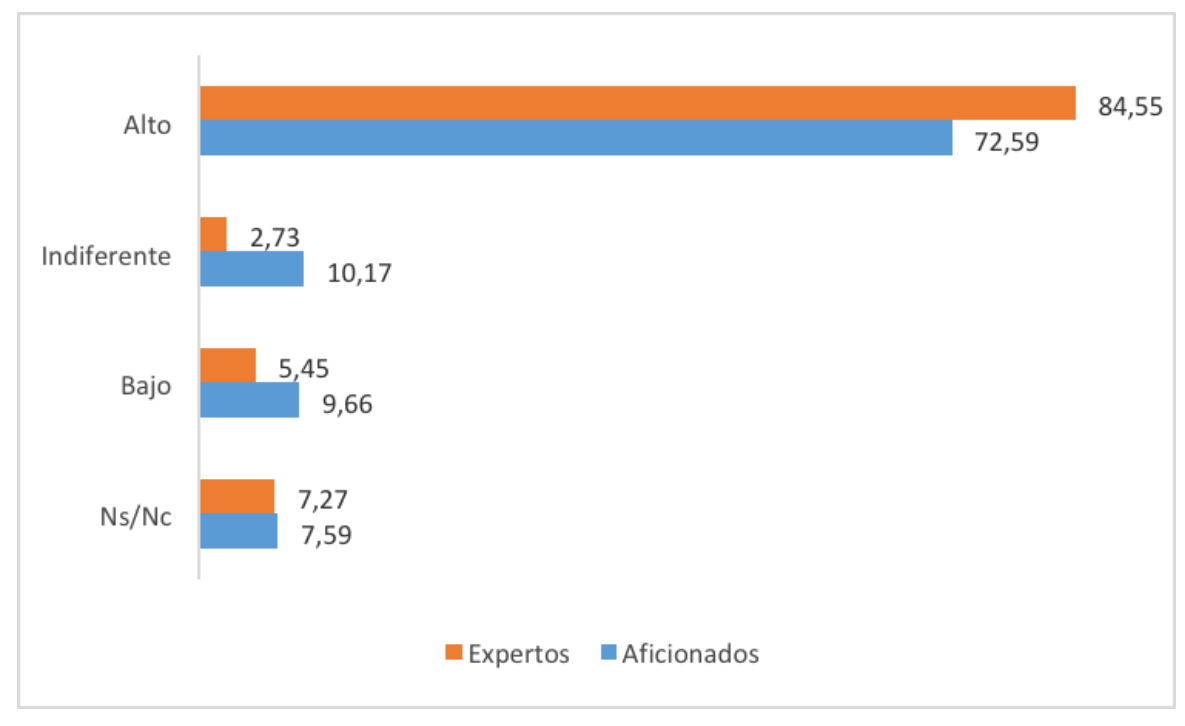

Gráfico 1. Valoración de la importancia de la interpretación. Fuente: Elaboración propia

En el caso de la valoración de la improvisación, se observa como la valoración de esta es alta por parte de ambas categorías. Concretamente más de un 84\% de los expertos y un $83 \%$ de los aficionados perciben que la aportación de valor cultural de los elementos ligados a la improvisación, como pueden ser la espontaneidad y la intuición del artista, es alto. 


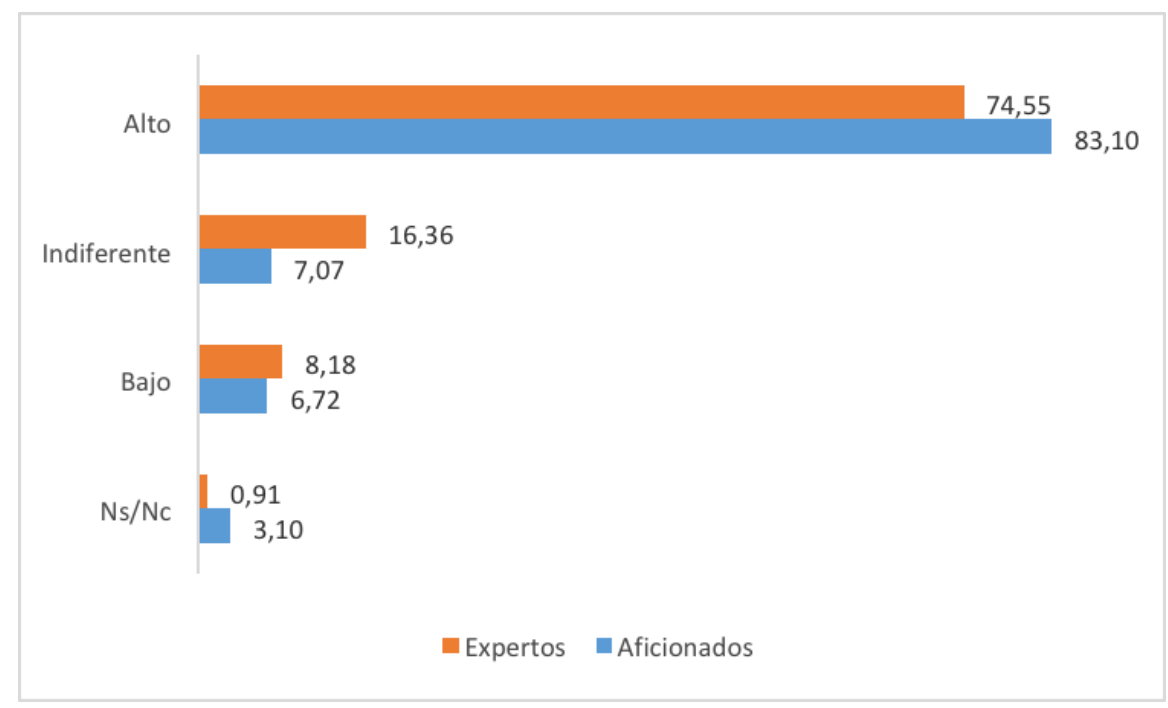

Gráfico 2. Valoración de la importancia de la improvisación.

Con estos resultados se demuestra empíricamente la brecha existente entre la percepción del valor cultural de la interpretación y los elementos ligados a la improvisación por parte de aficionados y expertos y la protección que reciben por parte de los diferentes ordenamientos jurídicos internacionales en materia de propiedad intelectual. 


\section{Conclusiones}

El artículo presenta para la música popular tradicional, concretamente para el flamenco, la brecha existente entre importancia de la interpretación en el proceso de creación y la protección que recibe por parte de los derechos de propiedad intelectual.

Para ello se explican las fuentes de creatividad que dan lugar a la obra: acervo cultural, composición e interpretación. A partir de dicha estructura se realiza una extensa revisión bibliográfica en tres disciplinas: artes escénicas, derecho y economía de la cultura sobre la interpretación. Con la revisión de la literatura de artes escénicas se pone de manifiesto la importancia de la interpretación y la idea de que en géneros musicales con tradición oral, la obra musical constituye un trabajo colaborativo entre autor e intérprete. La revisión de las legislaciones de propiedad intelectual ha permitido conocer el tratamiento que recibe y cuáles son los requisitos que piden los diferentes ordenamientos para protegerla con derechos de autor. Dichas legislaciones parecen suponer distintos tipos o niveles de aportaciones creativas a la obra final, provocando un desequilibrio en cuanto a protección en el mercado que afecta negativamente a la interpretación. Y, por último, la revisión de la literatura de economía de la cultura ha sido clave para diseñar una metodología basada en entrevistas y encuestas que pueda medir la importancia de la interpretación, a partir del concepto de valor cultural percibido.

Con las entrevistas se ha puesto de manifiesto que la interpretación imprime características distintivas a la obra musical que añaden valor a la misma y la hace reconocible para el público. Todos los entrevistados consideran la interpretación como un trabajo creativo y, por tanto, merece una protección equivalente a su importancia. 
Gracias a las encuestas se ha dejado patente la alta valoración que recibe la interpretación y los elementos ligados a la improvisación (espontaneidad e intuición artística) dentro de la obra musical por parte de dos categorías de agentes del mercado (aficionados y expertos). Los datos permiten observar la brecha entre el valor percibido de la interpretación a la obra musical y la protección que recibe por parte de los derechos de autor.

Para finalizar con una posible solución, autores como Bercovitz (2017) o Towse (2007) plantean la posibilidad de considerar el talento del intérprete y el del autor como equivalentes. Esto en términos legales se traduce en la consideración enmarcada dentro de las joint works defendidas por las leyes norteamericanas o la obra en colaboración defendida por los ordenamientos europeos, como el español (art. 7.1 TRLPI) (Heredia-Carroza et al., 2019b). Esto tiene defensores como Elliot (2003), quien considera que desarrollar relaciones con artistas que graban puede conducir a esfuerzos de colaboración que producen un mayor éxito comercial y creativo. Queda la discusión abierta sobre posibles soluciones.

\section{Agradecimientos}

Los autores agradecen a los revisores y al equipo editorial de la Revista Kepes, especialmente a su Editor Gustavo Alberto Villa Carmona, por sus útiles comentarios y discusiones realizadas, así como a los expertos entrevistados, a las organizaciones que dieron a conocer la encuesta (Flama. La guía del flamenco, el Centro Andaluz de Documentación del Flamenco y el sitio web Promocionmusical.com) y a las personas que respondieron la encuesta.

El presente trabajo ha sido financiado por el Programa de Movilidad Académica entre Instituciones Asociadas de la Asociación Universitaria Iberoamericana de Posgrado (AUIP) (a J. H. C.). 


\section{Referencias}

Aguado, L.F., Palma, L., y Pulido Pavón, N. (2017). 50 años de economía de la cultura. Explorando sus raíces en la historia del pensamiento económico. Cuadernos de Economía, 36 (70), 197-225.

Aix Gracia, F. (2002) El arte flamenco como campo de producción cultural. Aproximación a sus aspectos sociales. Revista Andaluza de Ciencias Sociales, 1, 109-125.

Au, W.T., Ho, G., and Chan, K.W.C. (2016). An Empirical Investigation of the Arts Audience Experience Index. Empirical Studies of the Arts, 35(1), 27-46. Doi: $10.1177 / 0276237415625259$.

Bercovitz, R. (2017). Comentarios a la Ley de Propiedad Intelectual. Madrid: Tecnos, 2017.

Bermúdez S., and Pérez, J. (2009). Introduction: Spanish Popular Music Studies. Journal of Spanish Cultural Studies, 10(2), 127-133. Doi: 10.1080/14636200902990661.

Blacking, J. (1981). Making artistic Popular Music: the goal of true Folk. Popular Music, 1, 9-14.

Bourdieu, P. (1984). Distinction, Cambridge, MA: Harvard University Press.

Castilla, M. (2010). El Flamenco y los Derechos de Autor. Madrid: REUS.

Castillo Ballén, S. (2015). Modos de relación sintiente Bocetos hacia una perspectiva de interpretación como ruta metodológica para la indagación de subjetividades. Cuadernos de Música, Artes Visuales y Artes Escénicas, 10(1), 129-150.

Caves, R. (2003). Contracts between Art and commerce. The Journal of Economic Perspectives, 17(2), 73-84. Doi: 10.1257/089533003765888430 
Chadabe, J. (1999). The performer is us. Contemporary Music Review, 18(3), 25-30. Doi: 10.1080/07494469900640321.

Chan, M.K., Au, W.T., and Ying, C. (2017). Developing Validating a Theater Experience Scale. Empirical Studies of the Arts, 35(2), 169-193. Doi: $10.1177 / 0276237416662737$.

Chojnacka, E. (2001). L'interprete-la memoire du compositeur (the performerthe essence of the composer. Contemporary Music Review 20(1), 25-34. Doi: 10.1080/07494460100640041.

Circular 92, Copyright Law of the United States and Related Laws Contained in Title 17 of the United States Code. Dec. 2012. Recuperado de https://www.copyright.gov/title17/

Circular 96. Code of Federal Regulations, Title 37- Patents, Trademarks, and Copyrights, Copyright Office regulations codified in the Code of Federal Regulations (CFR). Recuperado de https://www.copyright.gov/title37/202/

Cruces Roldán, C. (2001). El flamenco como patrimonio. Anotaciones a la Declaración de la voz de la Niña de los Peines como bien de interés cultural, Sevilla: Bienal de Arte Flamenco.

de Román, R. (2003). Obras musicales, compositores, intérpretes y nuevas tecnologías. Madrid: REUS.

Deleuze, G. y Guattari, (2002). F. Mil mesetas: capitalismo y esquizofrenia (J. Vázquez Pérez, Trad.). Valencia, España: Pre-Textos.

Directive 2011/77/EU of the European Parliament and of the Council of 27 September 2011, amending Directive 2006/116EC on the term of protection of copyright and certain related rights. Recuperado de http:// eur-lex.europa.eu/legal-content/ES/TXT/HTML/?uri=CELEX:32011L0077y from $=\mathrm{EN}$ 
Directiva 2001/29/CE, de 22 de mayo - LCEur $\backslash 2001 \backslash 2153$. Derecho de autor. Armonización de determinados aspectos de los derechos de autor y derechos afines a los derechos de autor en la sociedad de la información

Donnier, P. (2011). Descripción multimedia del flamenco. ¿Un nuevo medio de transmisión oral?, en José Miguel Díaz-Báñez \& Francisco Javier Escobar Borrego (Eds.): Investigación y flamenco. Sevilla: Signatura, 83-95.

Duby, M. (2017). Improvisation unfolding: process, pattern, and prediction. The Journal of New Paradigm Research. Doi: 10.1080/0204027.2017.1345249.

Edge, B. (2010). Comedy improvisation on television: does it work? Comedy Studies, 1(1), 101-111. Doi: 10.1386/cost.1.1.101/1.

Edinborough, C. (2012). Form and Spontaneity: training fo improvisation in football and theatre. Theatre, Dance and Interpretación Training, 3(2), 199207.

Elliot, J.I. (2003). From partnership to profits: the role of cowriting with recording artists as a strategic plan for copyright exploitation. Popular Music and Society, 26(2), 169-181. Doi: 10.1080/0300776032000095503.

Felton, M.V. (1978). The economics of the creative arts: the case of the composer. Journal of Cultural Economics, 2(1), 41-61. Doi: 10.1007/BF02479731.

Fernández, G.Z. (2001). Derechos morales en interpretaciones o ejecuciones fijadas. Rev. Prop. Inmaterial, 3, 95.

Geiger, C. (2017). Copyright as an access right: Securing cultural participation through the protection of creators' interests. In Giblin, R. y Kimberlee, W. (Eds.), What if we could reimagine copyright? ANU Press, 73-109.

Giblin, R. (2017). Reimagining copyright's duration. In Giblin, R. y Kimberlee, W. (Eds.), What if we could reimagine copyright? ANU Press, 177-211. 
González, A. (2001). La noción de obra audiovisual en el derecho de autor. Revista de propiedad intelectual, 7, 9-70. Recuperado de http://moodle2. unid.edu.mx/dts_cursos_mdl/lic/CYTC/ALC/AM/10/Nocion.pdf

González Betancur, J.D. (2013). Investigar, crear, interpretar. Reconocimiento del trabajo de creación teatral como ejercicio investigativo. Cuadernos de Música, Artes Visuales y Artes Escénicas, 8(1), 41-58.

Gorton, D. and Östersjö, S. (2016). Choose your own adventure music: on the emergence of voice in musical collaboration. Contemporary Music Review, 35(6), 579-598. Doi: 10.1080/07494467.2016.1282596.

Guerrero, J. (2016). La música de proyección folclórica en Buenos Aires: una disquisición sobre la escena musical. Cuadernos de Música, Artes Visuales y Artes Escénicas, 11(2), 187-202.

Hadi Akdede, S., and Ogus Binatli, A. (2017). Analysis of attedance in Turkish State theatres: evidence of the Veblen effect? Empirical Studies of the Arts, 35(2), 230-246. Doi: 10.1177/0276237416661987.

Hagtvedt, H., Hagtvedt, R. and Patrick, V.M. (2008). The perception and evaluation of visual arts. Empirical Studies of the Arts, 26(2), 197-218.

Harkins, P. (2012). Extending the term: the gowers review and the campaign to increase the length of Copyright in sound recordings. Popular Music and Society, 35(5), 629-649. Doi: 10.1080/03007766.2012.709664.

Heredia-Carroza, J., Palma Martos, L. \& Aguado, L. F. (2017). Originalidad Subjetiva y Copyright. El caso del flamenco en España. Anduli. Revista Andaluza de Ciencias Sociales, 16, 175-194.

Heredia-Carroza, J., Palma Martos, L. \& Aguado, L. F. (2019a). Song, performance and authorship: The case of flamenco in Spain. Trames. A Journal of the Humanities and Social Sciences, 23 (73/68), 1, pp. 3-14. Doi: 10.3176/tr.2019.1.01. 
Heredia-Carroza, J., Palma Martos, L., \& Aguado, L. F., (2019b). Flamenco y Derechos de Autor. El caso de Camarón de la Isla. Arbor, 195(791), a496. Doi: https://doi.org/10.3989/arbor.2019.791n1009.

Heredia-Carroza, J. (2019). Flamenco Performer's PerceivedValue: Development of a Measurement Index. Scientific Annals of Economics and Business. Doi: http://dx.doi.org/10.2478/saeb-2019-0017.

Heredia-Carroza, J., Palma Martos, L. y Aguado, L.F. (2019c). Why does copyright ignore performers? The case of flamenco in Spain. Journal of Arts Management, Law and Society, 49(5): 347-364. Doi: 10.1080/10632921.2019.1646682.

Heredia-Carroza, J., Palma Martos, L. y Aguado, L.F. (2020). "How to measure intangible cultural heritage value? The case of flamenco in Spain. Empirical Studies of the Arts. https://doi.org/10.1177/0276237420907865

Hernando, E., and Campo, S. (2017). Does the Artist's Name Influence the Perceived Value of an Art Work? International Journal of Arts Management, 19 (2), 49-58.

Hertzman, M.A. (2009). A Brazilian counterweight: music, intellectual property and the African Diaspora in Rio de Janeiro (1910s-1930s). Journal of Latin American Studies, 41(4), 695-722.

Holbrook, M.B. (1999). Popular Appeal versus Expert Judgments of Motion Pictures. Journal of consumer research, 26(2), 144-155. Doi: $10.1086 / 209556$.

Icazuriaga, J., Cuadrado, M. and Miquel, M.J. (Septiembre 2016). Analysing Perceived Value, Satisfaction and Purchase Intention in the Music Industry. $7^{\text {th }}$ Vienna Music Business Research Days, Vienna. 
Infante, B. (1980). Orígenes de lo flamenco y secreto del cante jondo. Sevilla: Junta de Andalucía, Consejería de Cultura.

Klamer, A. (2003) A pragmatic view on values in economics. Journal of Economic Methodology, 10(2), 1-24. Doi: 10.1080/1350178032000071075.

Levinson, J. (2015). Musical Concerns, Essays in Philosophy of Music. Oxford: Oxford University Press.

Lumer, R. (1991). Peter Seeger and the attempt to revive the folk music process. PopularMusicandSociety, 15(1), 45-58.Doi:10.1080/03007769108591422.

Lundy, D.E., and Smith, J.L. (2017). It's Tough to be a Critic: Professional vs. Non-professional Music Judgment. Empirical Studies of the Arts, 35(2), 139-168. Doi: 10.1177/0276237416661989.

Manuel, P. (2006). The Saga of a Song: Authorship and Ownership in the Case of Guantanamera. Latin American Music Review, 27(2), 121-147. Doi: 10.1353/lat.2007.0007.

Manuel, P. (2010). Composition, Authorship and Ownership in Flamenco, Past and Present. Ethnomusicology, 54(1), 106-135.

McNeil, A. (2017). Seed ideas and creativity in Hindustani raga music: beyond the composition-improvisation dialectic. Ethnomusicology Forum. Doi: 10.1080/17411912.2017.1304230.

McQueen, H.L., and Peacok, A. (1995). Implementing performing rights. Journal of Cultural Economics, 19, 157-175.

Meiseberg, B. (2014). Trust the artist versus trust the tale: interpretación implications of talent and self-marketing in folk music. Journal of Cultural Economics, 38(1), 9-42. Doi: 10.1007/s10824-012-9196-0.

Monasterio Astobiza, A. (2017). ¿Qué es cultura para la economía de la cultura? Definiendo la cultura para crear modelos mensurables en 
economía cultural. Arbor, 193(783), a376. Doi: http://dx.doi.org/10.3989/ arbor.2017.783n1007.

Ordóñez Flores, E. (2011). La perpetua reinvención de la identidad de los géneros en el baile flamenco. Arte, individuo y sociedad, 23(1), 19-28. Doi: 10.5209/rev_ARIS.2011.v23.n1.2.

Osborne, R. (2017). Is equitable remuneration equitable? Performers' rights in the UK. Popular Music and Society, 40(5), 573-591. Doi: 10.1080/03007766.2017.1348660.

Palma, L., Palma, M.L., Rodríguez, A., Martín, J.L., and Cascajo, I. (2017). Live Flamenco in Spain: A Dynamic Analysis of Supply, with managerial implications. International Journal of Arts Management, 19(3), 58-70.

Palmer, C. (1996). Anatomy of interpretation: sources of musical expression. Music Perception, 13(3), 433-453.

Pearson, V. (2010). Authorship and improvisation: musical lost property. Contemporary Music Review, 29(4), 367-378. Doi: 10.1080/07494467.2010.587314.

Pérez Peña, Ó.A. Derecho de autor y cultura popular tradicional en América Latina y el Caribe (Copyright and Traditional Popular Culture in Latin America and the Caribbean) (July 30, 2018). La Propiedad Inmaterial $\mathrm{N}^{\circ}$ 25, Enero-Junio 2018. Recuperado de https://ssrn.com/abstract=3222933

Pitt, I.L. (2010). Superstars effects on royalty income in a performing rights organization. Journal of Cultural Economics, 34(3), 219-236. Doi: 10.1007/ s10824-010-9123-1.

Plant A. (1934). The economic aspect of copyright in books. Economica, 1(2), 167-195. 
Prouty, K.E. (2006). Orality, literacy, and mediating musical experience: rethinking oral tradition in the learning of jazz improvisation. Popular Music and Society, 29(3), 317-334. Doi: 10.1080/03007760600670372.

Pulido, N., Palma, L., and Borrero, D. (2016). Copyright regimes in the European Union. An analysis of the Directive about the harmonisation (2001) effectiveness. 19th International Conference on Cultural Economics. Universidad de Valladolid. Valladolid.

Radbourne, J., Johanson, K., Glow, H., and White, T. (2010). The Audience Experience: measuring quality in the performing arts. International Journal of Arts Management, 11(3), 16-29.

Rangel, H. (2011). La observancia de los derechos de propiedad intelectual. Recuperado de http://www.wipo.int/edocs/pubdocs/es/intproperty/627/ wipo_pub_627.pdf

Real Decreto Legislativo 1/1996, de 12 de abril, por el que se aprueba el texto refundido de la Ley de Propiedad Intelectual, regularizando, aclarando y armonizando las disposiciones legales vigentes sobre la materia. Recuperado de http://noticias.juridicas.com/base_datos/Admin/rdleg1-1996.html

Reyes, J. (2013a) Canciones de máquina, los roles del intérprete y el instrumento musical. Encuentro de Estética y Nuevos Medios, Instituto Tecnológico de Medellín. Recuperado de https://ccrma.stanford.edu/ juanig/papers/ machine.pdf

Reyes, J. (2013b). Umbrales, intérpretes e interfaces en la ejecución de obras abiertas. Revista Ojo al Arte, ISSN: 1909-9134, Memorias Congreso Internacional de Artes del Caribe, Cartagena, Colombia. Recuperado de: https://ccrma.stanford.edu/ juanig/papers/threshold.pdf

Romero, J. (1996). La otra historia del flamenco. Sevilla: Junta de Andalucía. 
Rosón, T. (2010). El flamenco como obra musical, coreográfica y escénica. Las obras originales y derivadas: versiones, arreglos y utilización de las obras. In Castilla, M. (Ed.), El Flamenco y los Derechos de Autor, Madrid: REUS, 23-38.

Rushton, M. (1998). The moral Rights of Artists: Droit Moral ou Droit Pécuniaire? Journal of Cultural Economics, 22(1), 15-32. Doi: 10.1023/A:1007454719802.

San Cristóbal Opazo, U.P. (2018). ¿Acción, puesta en escena, evento o construcción audiovisual? Una breve introducción al concepto de interpretación en humanidades y en música. Cuadernos de Música, Artes Visuales y Artes Escénicas, 13(1), 207-231.

Schiaffini, G. (2006). Never improvise improvisation. Contemporary Music Review, 25(5/6), 575-576. Doi: 10.1080/07494460600990711.

Scionti, I. (2017). La guitarra flamenca: tradición e innovación (tesis doctoral). Universidad de Sevilla, Sevilla.

Sentencia de la Cour de Cassation, 1ère chambre civile - Manitas de Plata. 1 July 1970.

Steingress, G. (2007). Flamenco postmoderno: entre tradición y heterodoxia: un diagnóstico sociomusicológico (escritos 1989-2006). Signatura, Sevilla.

Thomas, P. (2007). Determining the indeterminate. Contemporary Music Review, 26(2), 129-140. Doi: 10.1080/07494460701250866.

Throsby, D. (2016). The Composer in the Market Place Revisited: The Economics of Music Composition Today. In Rizzo, I y Towse, R. The Artful Economist. A new look at Cultural Economics, Springer 153-170.

Towse, R. (2007). The Singer or the Song? Developments in Performers' Rights from the Perspective of a Cultural Economist. Review of Law and Economics, 3(3), 745-766. Doi:10.2202/1555-5879.1158. 
Webb, B. (2007). Partners in creation. Contemporary Music Review, 26(2), 255-281. Doi: 10.1080/07494460701250908.

Zavalloni, C. (2006). On improvisation. Contemporary Music Review, 25(5/6), 539-540. Doi: 10.1080/07494460600990638.

Como citar: Heredia-Carroza, J., Palma, L. y Aguado, L.F. (2020). Creación e interpretación en el marco de los derechos de autor. El caso de la música popular tradicional. Revista KEPES, 17 (21), 113-143. DOI: 10.17151/kepes.2020.17.21.5 